Article

\title{
Developing Gut Microbiota Exerts Colonisation Resistance to Clostridium (syn. Clostridioides) difficile in Piglets
}

\author{
Łukasz Grześkowiak*(D), Temesgen Hailemariam Dadi ${ }^{(D)}$, Jürgen Zentek and Wilfried Vahjen \\ Institute of Animal Nutrition, Freie Universität Berlin, Königin-Luise Strasse 49, 14195 Berlin, Germany \\ * Correspondence: lukasz.grzeskowiak@fu-berlin.de; Tel.: +49-30-838-52256
}

Received: 24 May 2019; Accepted: 15 July 2019; Published: 26 July 2019

\begin{abstract}
Clostridium (syn. Clostridioides) difficile is considered a pioneer colonizer and may cause gut infection in neonatal piglets. The aim of this study was to explore the microbiota-C. difficile associations in pigs. We used the DNA from the faeces of four sows collected during the periparturient period and from two to three of their piglets (collected weekly until nine weeks of age) for the determination of bacterial community composition (sequencing) and C. difficile concentration (qPCR). Furthermore, C. difficile-negative faeces were enriched in a growth medium, followed by qPCR to verify the presence of this bacterium. Clostridium-sensu-stricto-1 and Lactobacillus spp. predominated the gut microbiota of the sows and their offspring. C. difficile was detected at least once in the faeces of all sows during the entire sampling period, albeit at low concentrations. Suckling piglets harboured C. difficile in high concentrations (up to $\log _{10} 9.29$ copy number/g faeces), which gradually decreased as the piglets aged. Enrichment revealed the presence of $C$. difficile in previously $C$. difficile-negative sow and offspring faeces. In suckling piglets, the $C$. difficile level was negatively correlated with carbohydrate-fermenting bacteria, and it was positively associated with potential pathogens. Shannon and richness diversity indices were negatively associated with the $C$. difficile counts in suckling piglets. This study showed that gut microbiota seems to set conditions for colonisation resistance against $C$. difficile in the offspring. However, this conclusion requires further research to include host-specific factors.
\end{abstract}

Keywords: Clostridium difficile; Clostridioides; gut microbiota; sequencing; qPCR; enrichment; pig; suckling; weaned; sow

\section{Introduction}

The gut microbiota of pigs develops rapidly after birth, where the early gut colonisers between one and three days of age originate from the mother and environment [1]. This developing and yet fragile ecosystem is exposed to numerous environmental factors, which may shape its composition and activity until it becomes relatively more stable. The association between mother and offspring gut microbiota during early life is a critical factor for the subsequent succession of intestinal commensal bacteria, immune development, and gut digestive functions [2,3]. The early neonatal phase also seems to determine the microbial profile and intestinal health later in the offspring's life [4,5]. However, the aspect of mother-offspring association and its effect on early "microbial programming", including colonisation resistance to pathogens in pigs has not been studied in detail.

Clostridium (syn. Clostridioides) difficile is a spore-forming microorganism that inhabits the human and animal gut, including pigs, which are also potential reservoirs of this bacterium in the environment [6,7]. The shedding of this bacterium by farm animals is of concern regarding the zoonotic transmission to farm workers and food products [8,9]. C. difficile is one of the pioneer colonisers in neonatal piglets. C. difficile and its toxins are occasionally detected in gestating and lactating sows [10], 
indicating that the sows could most likely be a primary source of $C$. difficile spores for their offspring, including virulent ribotypes [11,12]. However, this has yet to be verified. Indeed, virulent ribotypes of C. difficile and its toxins are commonly detected in the faeces of new-born piglets $[9,10]$, where toxins may lead to spontaneous gut infection in neonatal piglets. However, it is unclear why and under what conditions piglets get sick or get asymptomatically colonised by virulent ribotypes. High levels of C. difficile and their toxins have been found in neonatal piglets without demonstrating symptoms of infection [10]. As in humans, the pathogenesis of $C$. difficile infection (CDI) is most likely multifactorial, and thus, accompanied by co-infections with other pathogens or a predisposing intestinal dysbiosis caused by antimicrobials $[13,14]$. Interestingly, C. difficile is rarely detected, and infection has not been reported in older pigs, including sows $[10,12,15]$. It seems that any disruption of the natural colonisation process or perturbances of the intestinal ecosystem could increase the chances of piglets developing CDI. This is well established in humans suffering from CDI, where antibiotic treatment is considered the main cause of recurrent CDI $[13,14]$. The phenomenon of colonisation resistance and susceptibility to CDI in piglets still needs to be elucidated.

The aim of this study was to explore the possible associations between gut microbiota and C. difficile colonisation in sows and their offspring.

\section{Materials and Methods}

\subsection{Animals and Sampling}

The institutional and national guidelines for the care and use of animals were followed. The sampling of faecal material from sows, suckling (1-4 weeks old), and weaned piglets (5-9 weeks old) required no ethical approval. Furthermore, the weaned piglets belonged to a control group of another trial approved by the Landesamt für Gesundheit und Soziales, Berlin on the date 03 April 2018 (identification number G0042/18). This study was conducted in the experimental pig facilities at the Institute of Animal Nutrition at the Freie Universität Berlin in Berlin, Germany. The institute has a sow breeding facility and its piglets are regularly produced (German Landrace). Each sow and their piglets were individually housed in commercial flat-deck pens. Gestating sows were fed a standard gestating (per kg of dry matter: $11.9 \mathrm{MJ}$ of metabolisable energy, $16.0 \%$ crude protein, $7.5 \%$ crude fibre) and a standard lactating (13.0 MJ of metabolisable energy, $17.9 \%$ crude protein, $5.0 \%$ crude fibre) diet after farrowing, according to standard recommendations by the GfE 2006 [16]. At weaning, the piglets were separated from their mothers and placed in pens (two pigs/pen) based on the plan from another trial. All animals remained healthy during the sampling period.

Faecal samples from four sows were collected at two and one months, and one week before, and one week after birth. A total of 102 faecal samples from randomly chosen offspring (two to three piglets/sow) from the four sows were collected weekly until nine weeks of age.

\subsection{DNA Extraction}

DNA from faecal samples (0.2 g) was extracted using the QIAamp DNA Stool Mini Kit (Qiagen, Hilden, Germany) following the manufacturer's instructions. The extraction protocol was preceded by repeated bead-beating on a FastPrep-24 ${ }^{\mathrm{TM}} 5 \mathrm{G}$ homogeniser (MP Biomedicals, LLC, Santa Ana, CA, USA) to increase DNA extraction performance from spore-forming [17] and possibly the gram-positive bacteria.

\subsection{Sequencing and Computational Data Analysis}

DNA extracts were subjected to amplicon sequencing using an Illumina NextSeq500 sequencer (LGC, Berlin, Germany). Using a universal primer set, the 341F-785R V3-V4 region of the 16S rDNA was targeted and sequenced, resulting in 4.5 million sequence-read-pairs. The forward and reverse reads had a median length of 197 and 264 nucleotides, respectively. The forward and reverse reads were also combined using the BBMerge tool (version 34.48) [18]. The median length of the combined 
reads was 404 nucleotides. After demultiplexing, the resulting 16S-rDNA sequences of 102 samples were analysed using the QIIME2 pipeline [19] to determine the microbial community profiles of each sample. Specifically, quality control was performed using the DADA2 [20] routine within QIMME2. In the quality control process, chimeric sequences were removed, and regions of sequences with low-quality scores were truncated. Then, the exact amplicon sequence variants [21] and their respective counts in each sample were determined using DADA2. Sequence variants with total counts that were less than five sequences were excluded from further analysis as they could be contaminants. Such sequences were relevant to a maximum of five samples. To account for the uneven distribution of sequences within samples, normalisation was done through rearification [22]. After rearification, all samples were represented equally by 10,000 sequences to accommodate the sample with the lest number of sequences. Taxonomic assignment of the exact amplicon sequence variants was done using QIIME2's feature classifier [23,24] together with the SILVA SSU database [25] (release 132). Subsequent genus-level taxonomic profiles were generated based on the assignment of sequences and their corresponding counts. Further statistical analysis was done using RStudio [26] and KNIME [27]. Specifically, R packages: stats, ggtree, and Hmisc were used to perform principal component analysis (PCA), phylogenetic, and correlation analyses, respectively. Principal components were calculated via singular value decomposition of the data matrix as defined by promp method under the $\mathrm{R}$ package stats. Differences for the diversity indices were calculated using the Kruskal-Wallis $\mathrm{H}$ test (significance at $p \leq 0.05$ ), and associations between the $C$. difficile counts and microbiota abundance (correlations with significance $p<0.1$ and rho $>0.5$ were considered) and between microbial diversity indices were assessed using the Pearson's $r$ correlation analysis procedure $(n=31)$. Given the minimal abundance of sequences belonging to $C$. difficile, no associations could be made between the qPCR results and the $16 \mathrm{~S}$ rDNA sequencing-based results.

\section{4. $q P C R$ and Sample Enrichment}

DNA extracts were also subjected to qPCR analyses. For qPCR, primer sequences for the specific detection of the $C$. difficile $16 \mathrm{~S}$ rDNA included Cdiff-16S-1f (5'-TTGAGCGATTTACTTCGGTAAAGA-3') and Cdiff-16S-1r (5'-CCATCCTGTACTGGCTCACCT-3'). The master mix consisted of Brilliant II SYBR Green QPCR Master Mix with Low ROX (Stratagene, San Diego, CA, USA). An AriaMx Real-Time PCR (Agilent Technologies Inc., Santa Clara, CA, USA) was used for PCR amplification and fluorescent data collection. Genomic DNA extracted from the $C$. difficile was used to construct a calibration curve and it served as a positive control. A qPCR reaction mixture where the DNA template was replaced with deionised water served as a negative control. The amplification programme and data collection were performed as previously described in Reference [17].

Faecal samples from animals that were negative for $C$. difficile as assessed by qPCR (no $C_{\mathrm{t}}$ or distinct melting curve) were enriched in a growth medium composed of a brain-heart-infusion broth, yeast extract, L-cysteine, $0.1 \%$ sodium taurocholate, $25 \%$ cycloserine, and $1.6 \%$ cefoxitin. Faecal suspensions $(10 \% w / v)$ were inoculated into the medium, homogenised by mixing, and then incubated anaerobically at $37^{\circ} \mathrm{C}$ for $48 \mathrm{~h}$. After that, the DNA extraction from $0.2 \mathrm{~g}$ of each sample was followed by the qPCR procedure described above, to verify the presence of $C$. difficile.

\section{Results}

\subsection{Bacterial Community Development}

In the individual animal samples, we found between 20 and 120 bacterial taxa. The total number of identified bacterial taxa was 277 , of which the 25 dominant taxa were displayed in stacked bar plots. There were 252 non-specified bacterial taxa (Figure 1a).

Taxa of Clostridium sensu stricto 1 and Lactobacillus predominated the gut microbiota of the sows and their offspring throughout the sampling period. The sows gut microbiota was characterised by a high abundance of Clostridium sensu stricto 1, Lactobacillus spp., Bacteroidales, 
Christensenellaceae R7, Clostridiales, Streptococcus spp., Terrisporobacter spp., Blautia spp., Ruminococcaceae UCG005, E. coprostanoligenes, Romboulsia spp., and Turicibacter spp. In one-week-old piglets, Lactobacillus spp., Clostridium sensu stricto 1, Clostridiales, Streptococcus spp., Bacteroides spp., and Escherichia-Shigella taxa dominated the gut microbiota.

From the second week onwards, bacterial taxa belonging to Bacteroidales, Ruminococcaceae UCG 002, Christensenellaceae R7 and Romboutsia spp., Ruminococcaceae NK4A214, and Ruminococcus 2 spp. increased in abundance, whilst Escherichia-Shigella diminished. From the third week onwards, Christensenellaceae R7 increased in abundance, and from the fifth week onwards (beginning of weaning), taxa of Ruminococcus 2 spp. and Lachnoclostridium spp. diminished and Prevotellaceae NK3B31 appeared. In that week, bacterial taxa belonging to Clostridium sensu stricto 1 were not detected, whilst Escherichia-Shigella reappeared. Between the sixth and ninth weeks, the gut microbiota was dominated by Lactobacillus spp., Clostridium sensu stricto 1, Bacteroidales, Christensenellaceae R7, Clostridiales, Ruminococcaceae ae UCG002, Terrisporobacter spp., Blautia spp., Ruminococcaceae UCG005, E. coprostanoligenes, Ruminococcaceae NK4A214, and Coprococcus 3. The relative abundance of bacterial taxa in the sow and offspring faeces over time are shown in Figure $1 \mathrm{a}$.

The sows, suckling, and weaned piglets shared 135 bacterial taxa, whilst the sows and suckling piglets shared 11 taxa, the suckling and weaned piglets shared 30 taxa, and the weaned piglets and sows shared 36 taxa (Figure 1b). Sows microbiota was unique for Lachnospiraceae, Anaerococcus spp., and Lachnoclostridium spp. Suckling piglets were exclusively colonised by Actinomyces spp. and Actinobacillus spp., while Clostridium sensu stricto 6 and Anaerostipes spp. were only found in the weaned piglets. Common bacteria in the sows and suckling piglets mainly belonged to Peptostreptococcus spp., Erysipelatoclostridium spp., Luteococcus spp., Arcanobacterium spp., Corynebacterium spp., Succiniclasticum spp., Cerasicoccus spp., Pediococcus spp., Finegoldia spp., Kocuria spp., and Cutibacterium spp. Both the suckling and weaned piglets were colonised by Cloacibacillus spp. and Chlamydia spp., whilst the weaned piglets and sows shared microbiota belonging to Ruminococcaceae UCG 008 and Agathobacter spp. amongst others. Supplementary Table S1 shows a list of all the bacterial taxa that were unique and shared between sows, suckling, and weaned piglets.

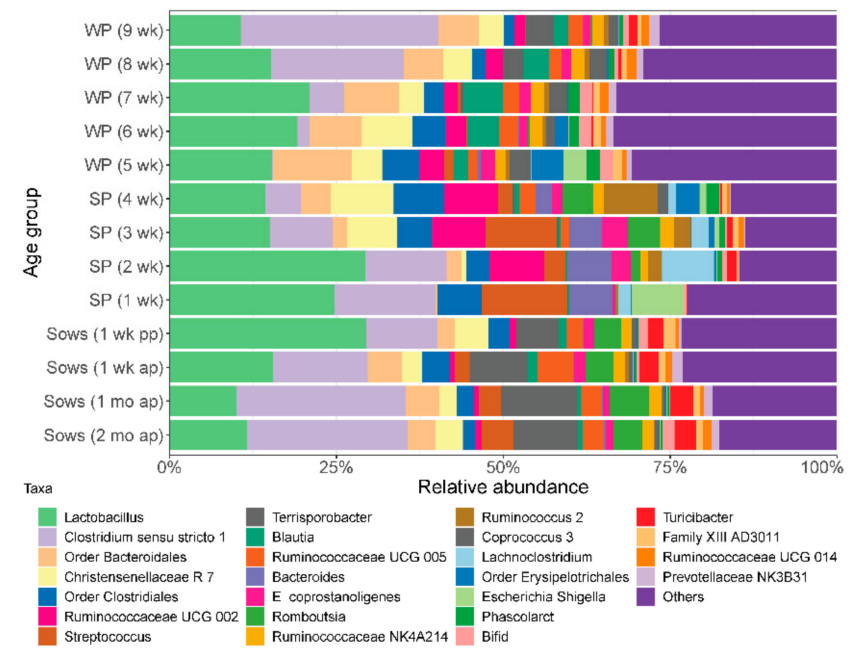

(a)

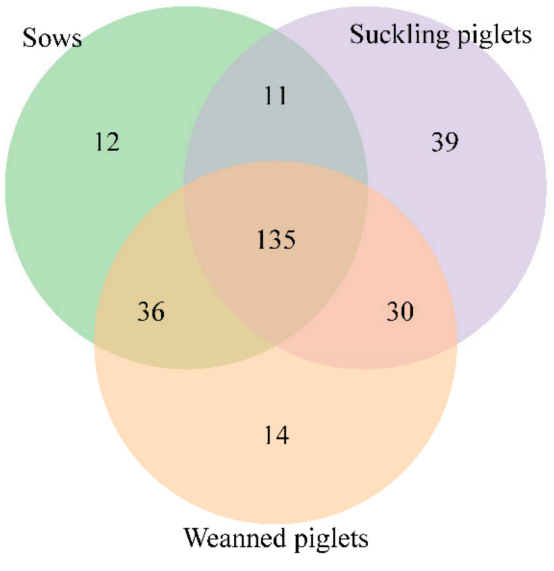

(b)

Figure 1. (a) Stacked bar plots showing the average percentage of bacterial taxa in sow and offspring faeces over time, (b) and a Venn diagram depicting the number of bacterial taxa that were unique and shared between sows, suckling, and weaned piglets, as analysed by the $16 \mathrm{~S}$ rDNA sequencing.

\subsection{Bacterial Diversity Analyses}

Microbial diversity as represented by richness, evenness, and the Shannon indices was lower in the suckling, but higher in the weaned piglets which were similar to those of the sows (Figure 2a-c). 
The PCA of the pig microbiome showed divergence by the age of the animals, clustering the weaned piglets closely with sows; whilst the suckling piglets were clustered more distantly from the weaned piglets and sows (Figure 2d). Comparison of the individual samples using the unweighted and weighted UniFrac distances showed the clustering by age groups (sows, suckling piglets, weaned piglets) (Supplementary Figure S1a,b).

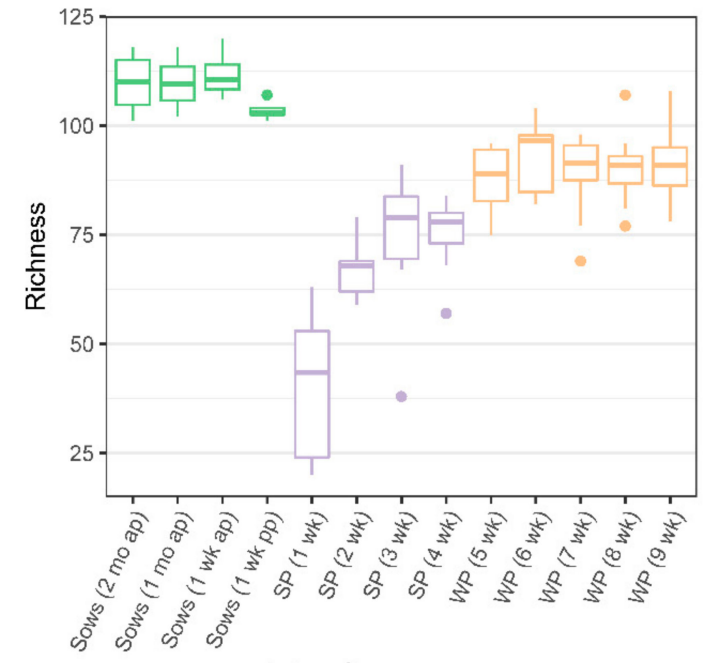

(a) Age group

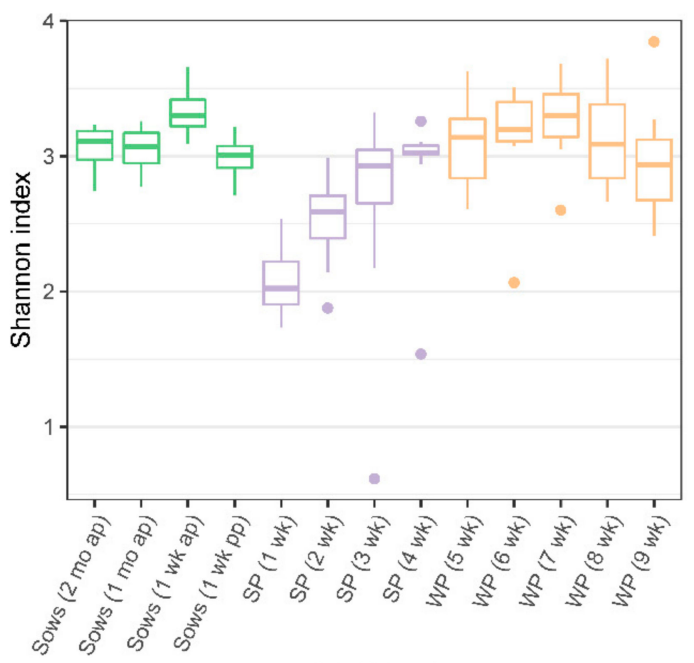

(c) Age group

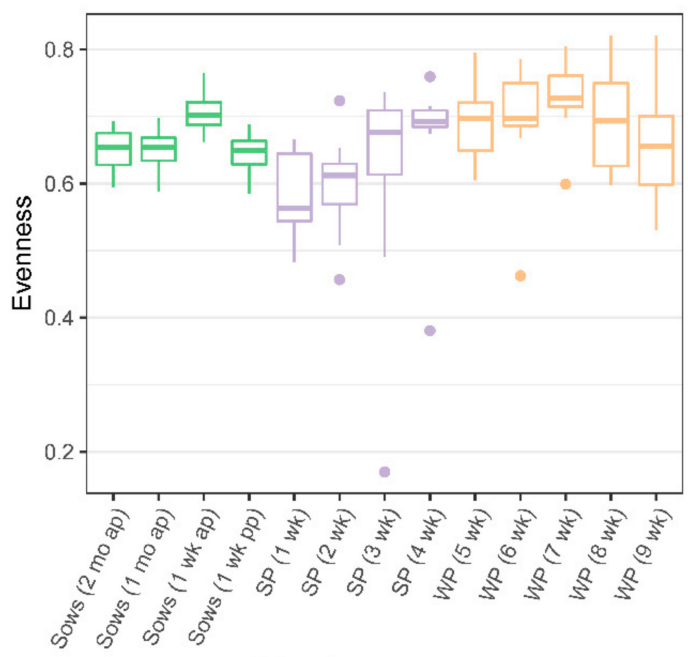

(b) Age group

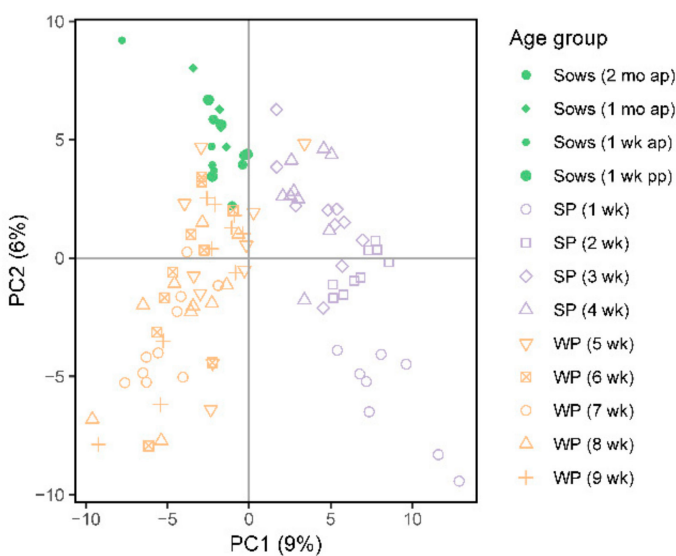

(d)

Figure 2. Diversity shown as (a) richness, (b) evenness, and (c) Shannon indices, and (d) a principal component analysis performed using the relative abundance of faecal microbial taxa in sows (green box plots), suckling (violet box plots) and weaned piglets (orange box plots) over time, as analysed by the $16 \mathrm{~S}$ rDNA sequencing. Dots in figures $(\mathbf{a}-\mathbf{c})$ indicate outliers. ap: antepartum; pp: postpartum.

\subsection{C. difficile Colonisation}

The presence of $C$. difficile was observed in each of the four sows during the sampling period, albeit at a low concentration (mean $\log _{10} 3.10 \pm 0.64$ copy number/g faeces). One month before farrowing, $C$. difficile was only found in one sow at a concentration of $\log _{10} 2.26$ copy number/g of faeces. Meanwhile, one week before farrowing, only one sow carried quantifiable $C$. difficile at a concentration of $\log _{10} 3.00$ copy number/g of faeces. Subsequently, one week after farrowing, two sows had C. difficile at a mean concentration of $\log _{10} 3.56 \pm 0.14$ copy number/g of faeces. Enrichment of $C$. difficile negative 
samples revealed the presence of this bacterium in two sows at two different sampling periods each, i.e., one month and one week before farrowing (Supplementary Table S2).

In the first two weeks of life, all piglets harboured C. difficile and at high concentrations up to $\log _{10} 9.29$ copy number/g faeces. The concentration of $C$. difficile gradually decreased as the piglets aged (Figure 3).

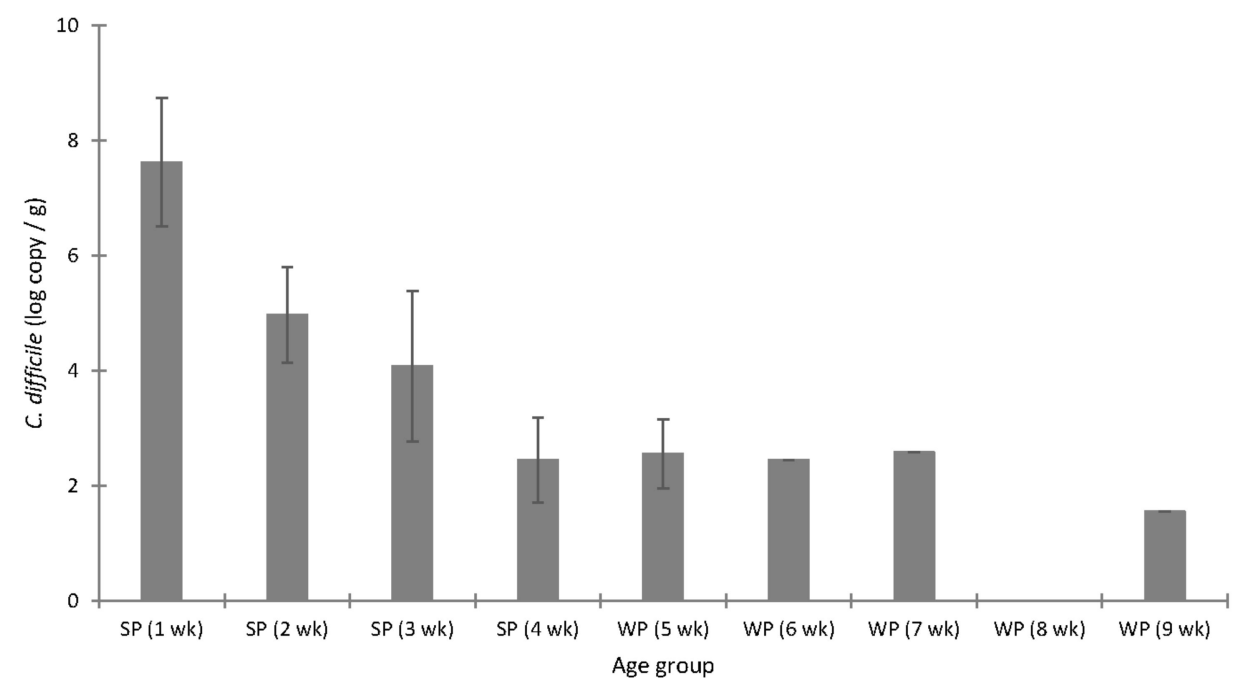

Figure 3. Concentration (mean $\pm \mathrm{SD}, \log _{10} \operatorname{copy} / \mathrm{g}$ ) of $C$. difficile in faecal samples from the offspring $(n=8-10)$ sampled weekly from one until nine weeks of age and analysed using qPCR, without previous sample enrichment. SP: suckling piglets; WP: weaned piglets.

C. difficile prevalence was $86 \%$ before and $97 \%$ after enrichment in the suckling piglets, and $12 \%$ before and $53 \%$ after enrichment in the weaned piglets. Overall, out of 48 offspring faecal samples that were negative for $C$. difficile from the first detection attempt using qPCR, a total of 23 turned positive $(48 \%)$ after enrichment in growth media followed by qPCR detection, confirming the presence of this species in the gut (Table 1).

Table 1. Prevalence (\%) of C. difficile in the faecal samples from the offspring (suckling and weaned piglets) $(n=8-10)$ sampled weekly from one until nine weeks of age. Faecal samples negative for C. difficile from qPCR were enriched in a specific growth media, followed by DNA extraction and qPCR.

\begin{tabular}{ccc}
\hline \multirow{2}{*}{ Age (Week) } & \multicolumn{2}{c}{ \% of Positive Samples } \\
\cline { 2 - 3 } & Before Enrichment & After Enrichment \\
\hline 1 & 100 & 100 \\
2 & 100 & 100 \\
3 & 90 & 90 \\
4 & 56 & 100 \\
5 & 30 & 80 \\
6 & 10 & 60 \\
7 & 10 & 60 \\
8 & 0 & 10 \\
9 & 11 & 56 \\
\hline
\end{tabular}

\subsection{Association of the Gut Microbiota and C. difficile in Suckling Piglets}

The correlation analyses revealed interactions of certain gut bacteria and C. difficile abundance in the suckling piglets. Notably, bacteria belonging to Ruminococcus 2 spp., Christensenellaceae R7, Ruminococcaceae UCG 002 and 010, Coprococcus 3 spp., and Prevotellaceae UCG 004 amongst others, were negatively associated with the level of $C$. difficile. On the other hand, bacterial taxa of Globicatella spp., Pasteurella spp., Clostridioides spp. (the majority of which is represented by C. difficile in SILVA SSU database), Clostridium sensu stricto 2, Peptostreptococcus spp., Enterococcus spp., Staphylococcus spp., and 
Moraxella spp. positively correlated with the concentration of $C$. difficile in suckling piglets (Table 2). Microbial diversity indices, such as Shannon and richness, were negatively associated with $C$. difficile counts in the suckling piglets ( $\mathrm{rho}=-0.4217, p=0.0181$ and rho $=-0.6093, p=0.0003$, respectively).

Table 2. Significant associations between the abundance of specific bacterial taxa (16S rDNA sequencing) and $C$. difficile counts (qPCR) in the faecal samples from suckling piglets that were sampled weekly from one until nine weeks of age.

\begin{tabular}{ccc}
\hline Taxon & Pearson's $\boldsymbol{r}(\boldsymbol{n}=\mathbf{3 1})$ & $p$ \\
\hline Ruminococcus 2 spp. & -0.5960 & 0.0004 \\
Christensenellaceae R7 & -0.5936 & 0.0004 \\
Romboutsia spp. & -0.5936 & 0.0004 \\
Marvinbryantia spp. & -0.5669 & 0.0009 \\
Ruminococcaceae UCG 002 & -0.5566 & 0.0012 \\
Fournierella spp. & -0.5488 & 0.0014 \\
Ruminococcaceae UCG 010 & -0.5296 & 0.0022 \\
Terrisporobacter spp. & -0.5286 & 0.0023 \\
Coprococcus 3 spp. & -0.5213 & 0.0026 \\
Hydrogenoanaerobacterium spp. & -0.5193 & 0.0028 \\
Prevotellaceae UCG 004 & -0.5188 & 0.0028 \\
Corynebacterium 1 spp. & -0.5163 & 0.0029 \\
Globicatella spp. & 0.5025 & 0.0040 \\
Pasteurella spp. & 0.5257 & 0.0024 \\
Clostridioides spp. & 0.5381 & 0.0018 \\
Clostridium sensu stricto 2 spp. & 0.5838 & 0.0006 \\
Peptostreptococcus spp. & 0.6064 & 0.0003 \\
Enterococcus spp. & 0.6272 & 0.0002 \\
Staphylococcus spp. & 0.6428 & $9.6402 \times 10^{-5}$ \\
Moraxella spp. & 0.6447 & $9.0628 \times 10^{-5}$ \\
\hline
\end{tabular}

\section{Discussion}

The gut microbiota is characterised by dynamic development after birth with certain bacteria temporarily dominating the intestinal tract. Using molecular tools, we followed the gut microbial succession and monitored colonisation development of $C$. difficile in pigs from the first until the ninth week of life. Notably, we focused on microbiota-C. difficile interrelations with regard to the phenomenon termed "colonisation resistance".

We found that in the first week of life, the gut microbiota was relatively simple and dominated by bacteria belonging to Lactobacillus spp., Clostridium sensu stricto 1 spp., Streptococcus spp., Bacteroides spp., and Escherichia-Shigella taxa. Although suckling piglets only shared a few bacterial taxa with sows, it was most likely that these bacterial settlers originated from the mother sow, acquired from birth canal, skin, milk, and from the surrounding environment. Similar reports on the early establishment of gut microbiota in pigs have been previously observed, where the pioneer colonisers included clostridia, enterobacteria, enterococci, streptococci, and peptostreptococci; whereas, lactobacilli and other species became predominant afterwards [1,28,29]. Introduction of solid feed during weaning reshapes the establishment of the bacterial community that began during the suckling period, and it has been associated with high rates of diarrhoea and mortality, mainly due to the overgrowth of pathogenic enterobacteria $[5,29,30]$. Interestingly, the gut microbiota of suckling piglets was characterised by low diversity, indicating an immature and developing micro-ecosystem. Low microbial diversity may be one of the factors that contribute to the succession of gut pathogens in early life, such as C. difficile. Low microbial competition may open a niche for opportunistic pathogens and predispose to disease development and/or pathogen dissemination in the environment. In the present study, we found that suckling piglets were highly colonised by $C$. difficile with regards to concentration and prevalence. The most likely source of $C$. difficile for the offspring was the mother sow where $C$. difficile spores and their toxins were detected during the gestation and lactation periods $[9,10]$. Here, we also report that the sow faeces contained $C$. difficile. It appeared that a low level of $C$. difficile in sows was sufficient to 
colonise the piglet gut successfully. However, the environmental dissemination of $C$. difficile cannot be overruled [11,12].

As the piglets aged, the concentration, as well as the prevalence of $C$. difficile-positive piglets, gradually decreased and, at the same time, the microbial diversity increased. Similarly, a decrease in the shedding load of $C$. difficile with age could be observed in calves [31]. We also found that a decrease in the $C$. difficile level was inversely associated with microbial diversity indices in suckling piglets. Specifically, $C$. difficile counts were negatively correlated with an increase in the abundance of bacteria specialised in carbohydrate degradation and short-chain-fatty-acid production in the hindgut of suckling piglets. Since all the piglets were colonised by $C$. difficile during the first two weeks of life, it was not possible to observe divergent microbiota development patterns in the animals that were colonised versus the animals that were not colonised by C. difficile. These results agreed with our previous observations regarding a very short time frame for colonisation by $C$. difficile, including toxigenic ribotypes [10]. Moreover, these data are very interesting and support the fact that microbial diversity is one of the factors that protects the host from $C$. difficile outgrowth and the risk for infection development. A phenomenon termed "colonisation resistance," where other bacteria in the developing ecosystem replace $C$. difficile could contribute to the primary protection of the host from CDI. Interestingly, it has been shown in humans that Clostridium scindens can outcompete C. difficile and prevent or ameliorate CDI [32]. Moreover, non-pathogenic or less virulent $C$. difficile ribotypes, which are natural colonisers of neonatal piglets, may successfully outcompete the toxigenic ribotypes, as we have previously observed in co-culture in vitro [33], and where other authors have observed in pigs and hamsters [34,35]. In addition, increased competition for nutrients accompanying the production of short chain fatty acids by carbohydrate-fermenting commensal microbiota could have a negative impact on the succession of $C$. difficile in the hindgut, as was demonstrated in a previous in vitro study [36]. On the other hand, any disruption to the natural colonisation process, for example, by introducing formula feeding or antibiotic therapy has been linked with lower microbial diversity and CDI development in both piglets and humans $[37,38]$. Therefore, undisturbed colonisation with the commensal microbiota could provide an effective first line of defence against opportunistic pathogens such as $C$. difficile. However, the successful colonisation by toxigenic $C$. difficile may lead to gut intoxication and CDI development in some piglets. Interestingly, not all piglets containing a high concentration of toxins in the gut will develop CDI [10]. The reasons for spontaneous outbreaks of CDI in piglets are mostly unknown. The CDI aetiology is most likely multifactorial, and the contribution of other pathogens to the infection development or host-specific factors cannot be ignored. We have found that the level of C. difficile was positively associated with the bacteria of Staphylococcus spp., Globicatella spp., Pasteurella spp., or Peptostreptococcus spp. amongst others, where the species strains are known swine pathogens. Therefore, infection with $C$. difficile could contribute to the development of co-infections by opening new niches to cohabiting pathogens. On the other hand, pathogenic bacteria could indirectly favour the growth of $C$. difficile by nutrient release from damaged gut tissues during infection processes. However, these scenarios still need to be explored in more detail. Nonetheless, as a host-specific factor, sufficient antibody titres against toxins transferred from the mother sow to the offspring through milk may be crucial in the protection against gut intoxication and CDI development $[33,39,40]$ once $C$. difficile colonisation is established in the gut.

Notably, almost half of the weaned-piglet-faeces that were negative for C. difficile (detected by qPCR) turned out to be positive after sample enrichment. The differences in $C$. difficile prevalence before and after sample enrichment indicated that this bacterium, although often reported as being absent in biological specimens, may still inhabit the host and shed spores into the environment. This may be a serious threat to the animals, farm workers, and the environment, in terms of antibiotic multi-resistance and dissemination of hyper-virulent ribotypes. Such findings are significant for the veterinary and clinical diagnostics of $C$. difficile, and they indicate that the prevalence of this bacterium may be underestimated when detection is performed without the sample enrichment medium that was used in this study. Our enrichment medium consisted of brain-heart-infusion, yeast extract, L-cysteine, 
sodium taurocholate for the induction of spore germination, and the antibiotics cycloserine and cefoxitin, that are known to inhibit a vast number of gram-positive and gram-negative bacteria without having an impact on $C$. difficile [41]. This approach allowed us to propagate $C$. difficile to a sufficient number of vegetative cells that became detectable using the qPCR method, thereby turning seemingly negative samples into positive ones. Indeed, the qPCR method has been one of the principal molecular techniques used to detect and quantify the bacteria of interest in faecal samples. The majority of studies describing $C$. difficile detection by qPCR in clinical specimens have not applied the sample enrichment method. Moreover, clostridia form spores that are considered the most challenging regarding nucleic acid extraction and molecular quantification of spore-forming bacteria. We have already successfully improved the DNA extraction method from spore-forming bacteria, such as C. difficile, and were able to decrease the detection limit by a log unit, compared to standard DNA extraction methods [17]. This technique improvement was applied in the present study to extract the microbial DNA for sequencing and $\mathrm{qPCR}$, and it significantly improved the signals from bacterial genomes. Indeed, nucleic acid extraction without special treatment for bacterial spores may underestimate the $C$. difficile cell numbers.

\section{Conclusions}

This study shows that the gut microbiota seems to set conditions for colonisation resistance against C. difficile in the offspring. However, additional host-factors cannot be overruled. The phenomenon of colonisation resistance and susceptibility to $C$. difficile infection in suckling piglets requires more focus. In the future, the modulation of gut microbiota could be an attractive approach to controlling $C$. difficile colonisation in piglets.

Supplementary Materials: The following are available online at http://www.mdpi.com/2076-2607/7/8/218/s1. Table S1. List of bacterial taxa that are unique and shared between sows, suckling and weaned piglets, as analysed by $16 \mathrm{~S}$ rDNA sequencing. Table S2. Concentration of $C$. difficile in faecal samples from the sows sampled during the periparturient period and analysed using qPCR without and with previous sample enrichment (faecal samples negative for $C$. difficile from qPCR were enriched in a specific growth media, followed by DNA extraction and qPCR). Figure S1. UniFrac (a) unweighted and (b) weighted phylogenetic distance analyses of the faecal microbial taxa in sows, suckling and weaned piglets over time, as analysed by $16 \mathrm{~S}$ rDNA sequencing. wk: week; mo: month; ap: antepartum; pp: postpartum.

Author Contributions: Conceptualisation, Ł.G., J.Z., and W.V.; Methodology, Ł.G. and T.H.D.; Software, T.H.D.; Formal analysis, Ł.G. and T.H.D.; Resources, J.Z. and W.V.; Data curation, Ł.G. and T.H.D.; Writing—original draft preparation, Ł.G. and T.H.D.; Writing—review and editing, J.Z. and W.V.; Visualization, Ł.G. and T.H.D.; Funding acquisition, Ł.G., J.Z., and W.V.

Funding: This study was financially supported by the German Research Foundation (Deutsche Forschungsgemeinschaft, DFG) grant GR 5107/2-1.

Acknowledgments: The publication of this article was funded by Freie Universität Berlin. We would like to thank B. Martinez-Vallespin and the animal caretakers for help in sample collection and sample availability, as well as M. Kochan for help in DNA extraction and qPCR.

Conflicts of Interest: The authors declare no conflict of interest.

\section{References}

1. Bian, G.; Ma, S.; Zhu, Z.; Su, Y.; Zoetendal, E.G.; Mackie, R.; Liu, J.; Mu, C.; Huang, R.; Smidt, H. Age, introduction of solid feed and weaning are more important determinants of gut bacterial succession in piglets than breed and nursing mother as revealed by a reciprocal cross-fostering model. Environ. Microbiol. 2016, 18, 1566-1577. [CrossRef] [PubMed]

2. Grönlund, M.; Grzeskowiak, Ł.; Isolauri, E.; Salminen, S. Influence of mother's intestinal microbiota on gut colonization in the infant. Gut Microbes 2011, 2, 1-7. [CrossRef] [PubMed]

3. Scharek-Tedin, L.; Kreuzer-Redmer, S.; Twardziok, S.O.; Siepert, B.; Klopfleisch, R.; Tedin, K.; Zentek, J.; Pieper, R. Probiotic Treatment Decreases the Number of CD14-Expressing Cells in Porcine Milk Which Correlates with Several Intestinal Immune Parameters in the Piglets. Front. Immunol. 2015, 6, 1-10. [CrossRef] [PubMed] 
4. Dou, S.; Gadonna-Widehem, P.; Rome, V.; Hamoudi, D.; Rhazi, L.; Lakhal, L.; Larcher, T.; Bahi-Jaber, N.; Pinon-Quintana, A.; Guyonvarch, A.; et al. Characterisation of Early-Life Fecal Microbiota in Susceptible and Healthy Pigs to Post-Weaning Diarrhoea. PLoS ONE 2017, 12, e0169851. [CrossRef] [PubMed]

5. Thompson, C.L.; Wang, B.; Holmes, A.J. The immediate environment during postnatal development has long-term impact on gut community structure in pigs. ISME J. 2008, 2, 739-748. [CrossRef] [PubMed]

6. Álvarez-Pérez, S.; Blanco, J.L.; Bouza, E.; Alba, P.; Gibert, X.; Maldonado, J.; García, M.E. Prevalence of Clostridium difficile in diarrhoeic and non-diarrhoeic piglets. Vet. Microbiol. 2009, 137, 302-305. [CrossRef] [PubMed]

7. Grześkowiak, L.; Collado, M.C.; Mangani, C.; Maleta, K.; Laitinen, K.; Ashorn, P.; Isolauri, E.; Salminen, S.; Maleta, K. Distinct Gut Microbiota in Southeastern African and Northern European Infants. J. Pediatr. Gastroenterol. Nutr. 2012, 54, 812-816. [CrossRef]

8. Rodriguez, C.; Taminiau, B.; Van Broeck, J.; Delmée, M.; Daube, G. Clostridium difficile in Food and Animals: A Comprehensive Review. Adv. Exp. Med. Biol. 2016, 932, 65-92. [PubMed]

9. Weese, J.S.; Wakeford, T.; Reid-Smith, R.; Rousseau, J.; Friendship, R. Longitudinal investigation of Clostridium difficile shedding in piglets. Anaerobe 2010, 16, 501-504. [CrossRef]

10. Grześkowiak, Ł.; Zentek, J.; Vahjen, W. Determination of the extent of Clostridium difficile colonisation and toxin accumulation in sows and neonatal piglets. Anaerobe 2016, 40, 5-9.

11. Hopman, N.; Keessen, E.; Harmanus, C.; Sanders, I.; Van Leengoed, L.; Kuijper, E.; Lipman, L. Acquisition of Clostridium difficile by piglets. Vet. Microbiol. 2011, 149, 186-192. [CrossRef] [PubMed]

12. Keessen, E.; Donswijk, C.; Hol, S.; Hermanus, C.; Kuijper, E.; Lipman, L. Aerial dissemination of Clostridium difficile on a pig farm and its environment. Environ. Res. 2011, 111, 1027-1032. [CrossRef] [PubMed]

13. Antharam, V.C.; Li, E.C.; Ishmael, A.; Sharma, A.; Mai, V.; Rand, K.H.; Wang, G.P. Intestinal Dysbiosis and Depletion of Butyrogenic Bacteria in Clostridium difficile Infection and Nosocomial Diarrhea. J. Clin. Microbiol. 2013, 51, 2884-2892. [CrossRef] [PubMed]

14. Schubert, A.M.; Rogers, M.A.M.; Ring, C.; Mogle, J.; Petrosino, J.P.; Young, V.B.; Aronoff, D.M.; Schloss, P.D. Microbiome Data Distinguish Patients with Clostridium difficile Infection and Non-C. difficile-Associated Diarrhea from Healthy Controls. mBio 2014, 5, e01021-14. [CrossRef] [PubMed]

15. Krůtová, M.; Zouharova, M.; Matejkova, J.; Tkadlec, J.; Krejci, J.; Faldyna, M.; Nyc, O.; Bernardy, J. The emergence of Clostridium difficile PCR ribotype 078 in piglets in the Czech Republic clusters with Clostridium difficile PCR ribotype 078 isolates from Germany, Japan and Taiwan. Int. J. Med. Microbiol. 2018, 308, 770-775. [CrossRef] [PubMed]

16. DLG Verlag. GfE Empfehlungen Zur Energie-Und Nährstoffversorgung Von Schweinen; DLG Verlag: Frankfurt, Germany, 2006.

17. Zentek, J.; Vahjen, W.; Grześkowiak, L. Physical Pre-Treatment Improves Efficient DNA Extraction and qPCR Sensitivity from Clostridium difficile Spores in Faecal Swine Specimens. Curr. Microbiol. 2016, 73, 727-731.

18. Bushnell, B.; Rood, J.; Singer, E. BBMerge-Accurate paired shotgun read merging via overlap. PLoS ONE 2017, 12, e0185056. [CrossRef]

19. Bolyen, E.; Rideout, J.R.; Dillon, M.R.; Bokulich, N.A.; Abnet, C.; Al-Ghalith, G.A.; Alexander, H.; Alm, E.J.; Arumugam, M.; Asnicar, F.; et al. QIIME 2: Reproducible, interactive, scalable, and extensible microbiome data science. PeerJ Prepr. 2018, 6, e27295v2. [CrossRef]

20. Callahan, B.J.; McMurdie, P.J.; Rosen, M.J.; Han, A.W.; A Johnson, A.J.; Holmes, S.P. DADA2: High-resolution sample inference from Illumina amplicon data. Nat. Methods 2016, 13, 581-583. [CrossRef]

21. Callahan, B.J.; McMurdie, P.J.; Holmes, S.P. Exact sequence variants should replace operational taxonomic units in marker-gene data analysis. ISME J. 2017, 11, 2639-2643. [CrossRef]

22. Weiss, S.; Xu, Z.Z.; Peddada, S.; Amir, A.; Bittinger, K.; González, A.; Lozupone, C.; Zaneveld, J.R.; Vázquez-Baeza, Y.; Birmingham, A.; et al. Normalization and microbial differential abundance strategies depend upon data characteristics. Microbiome 2017, 5, 27. [CrossRef] [PubMed]

23. Bokulich, N.A.; Kaehler, B.D.; Rideout, J.R.; Dillon, M.; Bolyen, E.; Knight, R.; Huttley, G.A.; Caporaso, J.G. Optimizing taxonomic classification of marker-gene amplicon sequences with QIIME 2's q2-feature-classifier plugin. Microbiome 2018, 6, 90. [CrossRef] [PubMed]

24. Pedregosa, F.; Varoquaux, G.; Gramfort, A.; Michel, V.; Thirion, B.; Grisel, O.; Blondel, M.; Prettenhofer, P.; Weiss, R.; Dubourg, V.; et al. Scikit-learn: Machine learning in Python. J. Mach. Learn. Res. 2011, 12, $2825-2830$. 
25. Yilmaz, P.; Parfrey, L.W.; Yarza, P.; Gerken, J.; Pruesse, E.; Quast, C.; Schweer, T.; Peplies, J.; Ludwig, W.; Glöckner, F.O. The SILVA and “All-species Living Tree Project (LTP)" taxonomic frameworks. Nucleic Acids Res. 2014, 42, D643-D648. [CrossRef] [PubMed]

26. RStudio Team. RStudio: Integrated Development for R; v0.98.1074; RStudio, Inc.: Boston, MA, USA, 2015.

27. Berthold, M.R.; Cebron, N.; Dill, F.; Gabriel, T.R.; Kötter, T.; Meinl, T.; Ohl, P.; Thiel, K.; Wiswedel, B. KNIME-The Konstanz Information Miner: Version 2.0 and Beyond. SIGKDD Explor. Newsl. 2009, 11, $26-31$. [CrossRef]

28. Chen, X.; Xu, J.; Ren, E.; Su, Y.; Zhu, W. Co-occurrence of early gut colonization in neonatal piglets with microbiota in the maternal and surrounding delivery environments. Anaerobe 2018, 49, 30-40. [CrossRef] [PubMed]

29. Frese, S.A.; Parker, K.; Calvert, C.C.; Mills, D.A. Diet shapes the gut microbiome of pigs during nursing and weaning. Microbiome 2015, 3, 1237439. [CrossRef]

30. Pluske, J.R.; Turpin, D.L.; Kim, J.C. Gastrointestinal tract (gut) health in the young pig. Anim. Nutr. 2018, 4, 187-196. [CrossRef]

31. Bandelj, P.; Harmanus, C.; Blagus, R.; Cotman, M.; Kuijper, E.J.; Ocepek, M.; Vengust, M. Quantification of Clostridioides (Clostridium) difficile in feces of calves of different age and determination of predominant Clostridioides difficile ribotype 033 relatedness and transmission between family dairy farms using multilocus variable-number tandem-repeat analysis. BMC Vet. Res. 2018, 14, 298.

32. Buffie, C.G.; Bucci, V.; Stein, R.R.; McKenney, P.T.; Ling, L.; Gobourne, A.; No, D.; Liu, H.; Kinnebrew, M.; Viale, A.; et al. Precision microbiome reconstitution restores bile acid mediated resistance to Clostridium difficile. Nature 2015, 517, 205-208. [CrossRef]

33. Grześkowiak, Ł.M.; Pieper, R.; Huynh, H.A.; Cutting, S.M.; Vahjen, W.; Zentek, J. Impact of early-life events on the susceptibility to Clostridium difficile colonisation and infection in the offspring of the pig. Gut Microbes 2018, 10, 251-259.

34. Nagaro, K.J.; Phillips, S.T.; Cheknis, A.K.; Sambol, S.P.; Zukowski, W.E.; Johnson, S.; Gerding, D.N. Nontoxigenic Clostridium difficile Protects Hamsters against Challenge with Historic and Epidemic Strains of Toxigenic BI/NAP1/027 C. difficile. Antimicrob. Agents Chemother. 2013, 57, 5266-5270. [CrossRef] [PubMed]

35. Songer, J.G.; Jones, R.; Anderson, M.A.; Barbara, A.J.; Post, K.W.; Trinh, H.T. Prevention of porcine Clostridium difficile-associated disease by competitive exclusion with nontoxigenic organisms. Veter. Microbiol. 2007, 124, 358-361. [CrossRef] [PubMed]

36. May, T.; Mackie, R.I.; Fahey, G.C.; Cremin, J.C.; Garleb, K.A. Effect of Fiber Source on Short-Chain Fatty Acid Production and on the Growth and Toxin Production by Clostridium difficile. Scand. J. Gastroenterol. 1994, 29, 916-922. [CrossRef] [PubMed]

37. Grześkowiak, Ł.; Martínez-Vallespín, B.; Dadi, T.H.; Radloff, J.; Amasheh, S.; Heinsen, F.A.; Franke, A.; Reinert, K.; Vahjen, W.; Zentek, J.; et al. Formula feeding predisposes neonatal piglets to Clostridium difficile gut infection. J. Infect. Dis. 2018, 217, 1442-1452. [CrossRef] [PubMed]

38. Elliott, B.; Chang, B.J.; Golledge, C.L.; Riley, T.V. Clostridium difficile-associated diarrhoea. Intern. Med. J. 2007, 37, 561-568. [CrossRef] [PubMed]

39. Kyne, L.; Warny, M.; Qamar, A.; Kelly, C.P. Association between antibody response to toxin A and protection against recurrent Clostridium difficile diarrhoea. Lancet 2001, 357, 189-193. [CrossRef]

40. Steele, J.; Mukherjee, J.; Parry, N.; Tzipori, S. Antibody against TcdB, but not TcdA, prevents development of gastrointestinal and systemic Clostridium difficile disease. J. Infect. Dis. 2013, 207, 323-330. [CrossRef]

41. Nerandzic, M.M.; Donskey, C.J. Effective and reduced-cost modified selective medium for isolation of Clostridium difficile. J. Clin. Microbiol. 2009, 47, 397-400. [CrossRef]

(C) 2019 by the authors. Licensee MDPI, Basel, Switzerland. This article is an open access article distributed under the terms and conditions of the Creative Commons Attribution (CC BY) license (http://creativecommons.org/licenses/by/4.0/). 